\section{Ergebnisse der Vorstandswahlen auf dem 97. RöKo}

Auf dem 97. Deutschen Röntgenkongress haben einige assoziierte Fachgesellschaften und Arbeitsgemeinschaften der DRG einen neuen Vorstand gewählt. Hier finden Sie eine Übersicht der neuen Vorstände:

Deutsche Gesellschaft für Interventionelle Therapie und minimal-invasive Therapie (DeGIR)

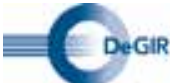

Vorsitzender:

Prof. Dr. Christian Stroszczynski, Universitätsklinikum Regensburg

Weitere Vorstandsmitglieder:

Prof. Dr. Ralf-Thorsten Hoffmann, Universitätsklinikum Carl-Gustav-Carus der TU Dresden

Prof. Dr. Peter Landwehr, DIAKOVERE Henriettenstift Hannover

Prof. Dr. Andreas Mahnken, Universitätsklinikum Marburg

Dr. Dominik Morhard, Leopoldina Krankenhaus Schweinfurt

Prof. Dr. Peter Reimer, Städtisches Klinikum Karlsruhe

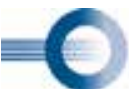

AG Gastrointestinal- und Abdominaldiagnostik

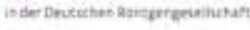

Vorsitzender:

Prof. Dr. Andreas G. Schreyer, MBA, Universitätsklinikum Regensburg

Stellvertretender Vorsitzender:

Prof. Dr. Lars Grenacher, Diagnostik München

Weitere Vorstandsmitglieder:

Prof. Dr. Markus Juchems, Klinikum Konstanz

Prof. Dr. Wieland H. Sommer, Klinikum Großhadern

Prof. Dr. Johannes Weßling, Clemenshospital Münster

AG Herz- und Cefábdiagnostik

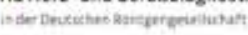

Vorsitzender:

Prof. Dr. Joachim Lotz, Universitätsklinikum Göttingen
Stellvertretende Vorsitzende:

Prof. Dr. Gabriele Krombach, Universitätsklinikum Gießen

Weitere Vorstandsmitglieder:

Prof. Dr. Matthias Gutberlet, Universität Leipzig

Prof. Dr. Fabian Bamberg, Universitätsklinikum Tübingen

Prof. Dr. Meinrad Beer, Universitätsklinikum Ulm

PD Dr. Katharina Fischbach, Otto von

Guericke Universität Magdeburg

Prof. Dr. David Maintz, Uniklinik Köln

Prof. Dr. Thomas Schlosser, Universitätsklinikum Essen

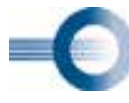

AG Informationstechnologie (@)

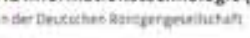

Vorsitzender:

Prof. Dr. Thomas Hackländer, Klinikum Wuppertal

Weitere Vorstandsmitglieder:

Prof. Dr. Elmar Kotter, Universitätsklinikum Freiburg

Dipl.-Phys. Roland Simmler, Hirslanden/

Zürich

PD Dr. Michael Walz, Ärztliche Stelle TÜV

Eschborn

Prof. Dr. Gerald Weisser, Uniklinik Mannheim

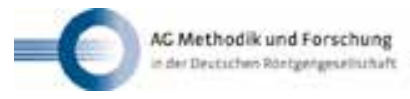

Vorsitzender:

Dr. Michel Eisenblätter, Universitätsklinikum Münster

Stellvertretender Vorsitzender:

PD Dr. Clemens Cyran, Klinikum der Universität München

Weitere Vorstandsmitglieder:

Prof. Dr. Fabian Bamberg, Universitätskli-

nikum Tübingen

PD Dr. Peter Bannas, Universitätsklinikum Hamburg-Eppendorf

Prof. Dr. Stefan Delorme, Deutsches Krebsforschungszentrum Heidelberg

Prof. Dr. Ingrid Hilger, Universitätsklinikum Jena
Prof. Dr. Fabian Kießling, Universitätsklinikum Aachen

Prof. Dr. Dr. Fritz Schick, Universitätsklinikum Tübingen

Prof. Dr. Matthias Taupitz, Charité - Universitätsmedizin Berlin

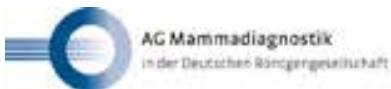

Vorsitzender:

Prof. Dr. Markus Müller-Schimpfle, Frankfurt-Höchst

Stellvertretender Vorsitzender: Prof. Dr. Walter Heindel, Münster Prof. Dr. Rüdiger Schulz-Wendtland, Erlangen

\section{Schriftführerin:}

PD Dr. Eva Maria Fallenberg, Berlin

Weitere Vorstandsmitglieder: PD Dr. Ute Kettritz, Berlin

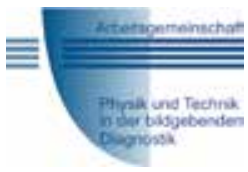

Vorsitzender:

Dr. Georg Stamm, Göttingen

Stellvertretender Vorsitzender:

Dipl.-Ing. Horst Lenzen, Köln

Weitere Vorstandsmitglieder: Dipl.-Ing. Olga Fröscher, Freiburg Dipl.-Phys. Roland Simmler, Aarau (CH) Dr. rer. nat. Jürgen Westhof, Kassel

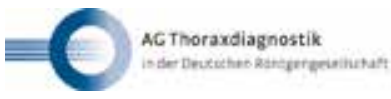

Vorsitzende:

PD Dr. Julia Ley-Zaporozhan, München PD Dr. Jens Vogel-Claussen, Hannover

Stellvertretender Vorsitzender: Prof. Dr. H.-U. Kauczor, Heidelberg

Schriftführer:

Prof. Dr. Jürgen Biederer, Heidelberg

Weitere Vorstandsmitglieder: Prof. Dr. Julien Dinkel, München Prof. Dr. Okka Hamer, Regensburg PD Dr. Hilmar Kühl, Essen PD Dr. Sebastian Ley, München Prof. Dr. Ulrich Müller-Lisse, München PD Dr. Dag Wormanns, Berlin 